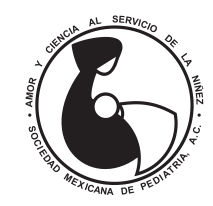

\title{
COVID-19: marca un antes y después en México, ¿punto y aparte?, ¿punto y seguido?
}

\author{
Carlos García-Bolaños, ${ }^{\ddagger} *$ Miguel Ángel Villasís-Keever ${ }^{\S}$ \\ ${ }^{\ddagger}$ Presidente de la Sociedad Mexicana de Pediatría; ${ }^{\S}$ Editor de la Revista Mexicana \\ de Pediatría, Instituto Mexicano del Seguro Social.
}

La propagación de la enfermedad denominada COVID-19 ha paralizado la vida normal en el mundo. Estamos viviendo un hecho inédito en las últimas décadas: una pandemia declarada por la OMS, que se originó en Wuhan, Hubei (China central) el pasado 01 de diciembre de 2019.

Como ya seguramente de todos es conocido, esta enfermedad es ocasionada por la infección del betacoronavirus SARS-CoV-2. El origen de este virus no es claro, pero parece que el ser humano lo contrajo a partir de un mamífero, probablemente un pangolín malayo. ${ }^{1}$ A lo largo de estos meses, hemos sido testigos de su elevada transmisibilidad por vía aérea, por lo cual rápidamente logró llegar a todos los países del mundo, sin importar razas, nivel socioeconómico, latitudes o temporada estacional. ${ }^{2}$

COVID-19 tiene una forma de presentación clínica variable. Por fortuna, lo más frecuente es la forma leve, donde los pacientes tienen poca o ninguna sintomatología, o bien, un cuadro similar a gripe (fiebre, tos, mialgia y astenia). Sin embargo, existen formas graves (5 al 20\% de los casos) con gran involucro pulmonar, lo que ocasiona insuficiencia respiratoria aguda grave, debido a infiltrado pulmonar bilateral que lleva a la muerte (2.3-3.8\%). En niños, las formas graves son menos frecuentes y las manifestaciones clínicas son

\footnotetext{
* Correspondencia: CGB, dr.garciabolanos64@gmail.com Conflicto de intereses: Los autores declaran que no tienen. Citar como: García-Bolaños C, Villasís-Keever MA. COVID-19: marca un antes y después en México, ¿punto y aparte?, ¿punto y seguido? Rev Mex Pediatr. 2020; 87(2):43-45 doi: 10.35366/94166 [COVID-19: It marks a before and after in Mexico, final point or period?]
}

generalmente más leves, aunque los lactantes pueden ser más vulnerables. ${ }^{3,4}$

En el mundo, España, Italia y Reino Unido resultaron devastados por la enfermedad, mientras que, en América, tanto Estados Unidos de Norteamérica (EUA) como Brasil, Perú y Ecuador tienen grandes problemas por la cantidad de pacientes detectados. ${ }^{2}$

Ha sido tan grande esta epidemia que políticos, artistas y deportistas de gran renombre han resultado contagiados. Entre otros, podemos mencionar a Boris Johnson (primer ministro de Inglaterra), la Reina Isabel II, o Angela Merkel (primer ministro de Alemania). En el ámbito a nivel nacional, el gobernador de Hidalgo y el Director General del Instituto Mexicano del Seguro Social (IMSS).

\section{La transformación de la vida de los mexicanos}

Desde el inicio de los contagios en México (primer caso confirmado: 27 febrero de 2020), y para tratar de contener la diseminación acelerada, los primeros días de marzo de 2020 el gobierno implementó diversas medidas como el lavado de manos y el aislamiento o distanciamiento social, así como el uso de cubrebocas. ${ }^{5}$ Posteriormente, para finales de marzo se cerraron de manera obligatoria tanto escuelas como centros de reunión multitudinaria: centros comerciales, teatros, cines, iglesias, gimnasios, entre otros. Además, se solicitó, en la manera de lo posible, cerrar centros de trabajo y fábricas "no esenciales", es decir, aquéllos espacios ajenos a la salud, alimentación, transporte, comunicación y gobierno. Incluso se implementaron medidas para disminuir el tránsito vehicular, como prohibir circular un día a la semana.

El eslogan que se ha utilizado ha sido: Quédate en casa, salva vidas, el cual ha sido difundido por todos los 
medios de comunicación, y para facilitar el mensaje se creó a Susana distancia, un personaje que promueve permanecer a más de un metro y medio de distancia entre personas, ${ }^{6} \mathrm{a}$ fin de evitar contagios.

Por todo lo anterior, cada ciudadano ha tenido que adaptarse a una nueva forma de vida, como trabajar en casa o a distancia para seguir laborando de manera activa. Asimismo, dado el cierre de las escuelas, se tuvo que recurrir a que, tanto alumnos de preescolar, escolar, secundaria, preparatoria y de universidad tuvieran que continuar el aprendizaje vía remota, ya sea por Internet o televisión abierta.

Los parques y restaurantes también han sido cerrados, por lo que las actividades sociales se han visto reducidas. De tal forma que se ha tenido que recurrir a cancelar o modificar las distintas celebraciones, como el viacrucis en Iztapalapa en Semana Santa, que este año fue llevado a cabo sin público por primera vez en 177 años. Lo mismo ocurrió con el Día del Niño o el Día de la Madre. Entonces, para evitar el contacto directo, para conmemorar estas fechas, las "reuniones" son virtuales, por videoconferencia a través de aplicaciones, ya sea por el teléfono celular o por computadoras. Amigos y amigas pasan buena parte del día enviando mensajes por redes sociales.

\section{Los efectos sociales y personales por COVID-19}

Han sido muchos días desde que inició la pandemia, aun así, persisten los incrédulos sobre la afectación que genera el COVID-19, por lo que piensan que no deben seguir las recomendaciones. Sólo el peligro de la muerte los ha obligado a cumplir las reglas de protección. Se han generado muertes irreparables, familias desconsoladas, proyectos de vida truncados. Al momento de escribir este texto, se tienen registradas más de 5 mil muertes en México, pero lamentablemente no están permitidas las ceremonias luctuosas ni entierros en cementerios, lo cual forma parte de las medidas de mitigación. En la medida de lo posible, los cuerpos deben ser cremados, los hornos trabajan a tope, pero cada muerto debe esperar su turno en una larga fila.

Asimismo, las visitas por parte de los familiares a los hospitales han sido restringidas al máximo, siendo mayor cuando se encuentran en terapia intensiva. De esta forma, algo inédito ha sido ser testigos de cómo la tecnología ha ayudado, ya que los informes y la comunicación entre familiares y pacientes se da por videollamadas.

Hay un vacío que, conforme pasan los días, tal parece que se engrosan las paredes de nuestras habitaciones y pone a prueba nuestra capacidad de resiliencia. No sabemos cuánto puede durar este encierro, por lo que estamos en incertidumbre sobre qué pasará en un futuro cercano. Los pensamientos son variados, por ejemplo, extrañamos la rutina, los horarios, los planes, el orden, la estructura para usar el tiempo. Lo cual resulta contradictorio sobre los deseos habituales; normalmente estamos esperando tener más tiempo para estar en nuestro hogar y, ahora que se nos concede, parece ser que no sabemos manejarlo.

\section{Repercusiones en el área médica por el COVID-19}

En un principio, las autoridades sanitarias expresaron: "es un fenómeno nuevo, pero parece ser moderado en su capacidad de producir enfermedad". No obstante, rápidamente surgieron varios aspectos relevantes. México se encontró con la realidad de no contar con suficientes médicos ni hospitales para hacer frente a esta emergencia sanitaria. Muchos hospitales a lo largo del país han sido reconvertidos, es decir, su funcionamiento solamente está enfocado a la atención de pacientes con COVID-19. Incluyendo hospitales de todo el Sector Salud, como Institutos Nacionales de Salud, hospitales públicos de los diferentes estados de la República Mexicana, así como del IMSS, ISSSTE, Secretaría de la Defensa Nacional y Marina. Asimismo, ha sido necesario contratar a más de 6 mil médicos generales y 12 mil enfermeras de manera temporal.

Un punto trascendente fue que evidentemente los insumos de salud sólo alcanzarían para el 5\% de enfermos por COVID-19. Por lo anterior, es indiscutible que era necesario el equipamiento de las unidades médicas; sin embargo, a lo largo del tiempo, se ha observado que las necesidades en distintas áreas han sido críticas de solucionar, por ejemplo, proporcionar los equipos de protección personal (EPP) a todo el sector de salud, como guantes, cubrebocas especiales, batas y caretas. En los primeros meses, ante la posibilidad de aumentar los contagios por falta de EPP, los alumnos de medicina y los internos de pregrado fueron excluidos de los hospitales.

Por otro lado, también se previó que los ventiladores para pacientes graves eran insuficientes, por lo que fue necesario adquirir alrededor de 10 mil ventiladores a los EUA y posteriormente a China.

Además, dada la conversión de hospitales, los privados firmaron un convenio con el Gobierno Federal para utilizar 3,300 de sus camas para atender pacientes sin COVID-19, para atender pacientes con problema agudos, como las cirugías para apendicitis, o bien, para la atención de partos y cesáreas. 
Obviamente, el equipo de salud ha sido un grupo vulnerable a lo largo de la pandemia. En cualquiera de los países, se ha contagiado tanto el personal médico como el de enfermería, camilleros, personal de intendencia, laboratoristas, llevando lamentablemente a algunos a la muerte. Alrededor del mundo se ha calculado que menos del 15\% del total de casos con COVID-19 corresponden a personal de salud. ${ }^{7}$ Por esta razón, el miedo al contagio, la saturación de los hospitales y observar diariamente la alta mortalidad en terapias intensivas llevan al desgaste físico y emocional, por lo que se ha convertido en una prioridad el cuidado de la salud mental del personal en hospitales COVID-19.8,9

Por si no fuera suficiente, de una manera cobarde e inexplicable (probablemente por miedo a lo desconocido), el personal de salud ha sufrido agresiones de una parte de la población civil, bajo el pretexto que constituyen riesgo para el contagio de los demás. Por fortuna, es mucho mayor la proporción de ciudadanos que agradecen al personal de salud, por estar arriesgando su vida en la primera línea "de fuego". Todos los días somos testigos de emotivos mensajes de aliento a través de testimonios, videos y memes. Pero también hay que agradecer a personas y agrupaciones privadas que se han organizado para realizar donativos a hospitales de todo tipo, incluyendo comida preparada y EPP.

\section{Acciones de la Sociedad Mexicana de Pediatría ante la epidemia}

La Sociedad Mexicana de Pediatría (SMP) temporalmente cierra sus puertas el 14 de marzo, con el fin de evitar contagios entre sus socios y el propio personal. Pero el trabajo del personal administrativo ha continuado desde casa, lo cual ha permitido seguir programando actividades de educación médica continua en línea, así como la publicación de la Revista Mexicana de Pediatría. Por su parte, la Mesa Directiva sesiona a través de una aplicación digital.

Ante la dificultad de llevar a cabo un evento de manera presencial, por primera vez se difiere nuestro magno Congreso Anual, que en esta ocasión se llevaría a cabo a finales del mes de abril y celebraría los 90 años de la fundación de nuestra querida SMP.

Como parte de su compromiso social con la comunidad de los trabajadores de la salud, la SMP realizó la donación de 1,000 equipos EPP a hospitales con carencias. Igualmente, emitimos cápsulas informativas y motivacionales, con el objetivo de palear la difícil situación que atraviesa el personal que opera en las distintas instituciones nacionales.

\section{Comentarios finales}

Ante la realidad que muy probablemente COVID-19 llegó para quedarse, y porque hasta ahora no disponemos de una vacuna para prevenir el avance de la enfermedad, las medidas de contención, la colaboración y el apoyo mutuo son las principales estrategias contra la pandemia.

Estamos orgullosos de la manera en que el equipo de salud, tanto en México como en el resto del mundo ha enfrentado este desafío. Como parte de lo aprendido, cuando hayamos superado esta etapa, tendremos que reflexionar sobre cómo fortalecer nuestro sistema de salud y nuestra capacidad de reacción para otras posibles pandemias.

Si bien, nunca hemos perdido la esperanza que en algún momento nos reencontraremos con amigos y familias, tenemos que reconocer que no debemos volver a la normalidad que teníamos antes de esta pandemia, ya que mucho de lo que acontece hoy es resultado de prácticas que debemos dejar en el pasado.

\section{REFERENCIAS}

1. Aragón-Nogales R, Vargas-Almanza I, Miranda-Novales MG. COVID-19 por SARS-CoV-2: la nueva emergencia de salud. Rev Mex Pediatr. 2019; 86(6): 213-218. doi: 10.35366/91871.

2. WHO Coronavirus Disease (COVID-19) Dashboard. Available in: https://covid19. who.int/?gclid=Cj0KCQjwirz3BRD_ ARIsAlmf7LNadJDZ-SdcAlyYDXvtRB4HG32qc8GYoubl2OpXx PQEppgKUd2OAVwaAv2QEALw_wcB.

3. Livingston E, Bucher K. Coronavirus disease 2019 (COVID-19) in Italy. JAMA. 2020; 323(14): 1335. doi: 10.1001/ jama.2020.4344.

4. Montaño-Luna VE, Pacheco-Rosas DO, Vázquez-Rosales JG, Labra-Zamora MG, Fuentes-Pacheco Y, Sámano-Aviña M et al. Manejo clínico de casos pediátricos de COVID-19. Disponible en: http://revistamedica.imss.gob.mx/editorial/index.php/revista_ medica/article/view/3641/3738.

5. Chu DK, AkI EA, Duda S, Solo K, Yaacoub S, Schünemann HJ et al. Physical distancing, face masks, and eye protection to prevent person-to-person transmission of SARS-CoV-2 and COVID-19: a systematic review and meta-analysis. [published online ahead of print, 2020 Jun 1]. Lancet. 2020. S0140-6736(20)31142-9. doi: 10.1016/S0140-6736(20)31142-9.

6. Gobierno de México. Disponible en: https://coronavirus.gob. $\mathrm{mx} /$.

7. Chou R, Dana T, Buckley DI, Selph S, Fu R, Totten AM. Epidemiology of and risk factors for coronavirus infection in health care workers: a living rapid review. Ann Intern Med. 2020; M201632. doi: 10.7326/M20-1632.

8. Brooks SK, Webster RK, Smith LE, Woodland L, Wessely S, Greenberg N et al. The psychological impact of quarantine and how to reduce it: rapid review of the evidence. Lancet. 2020; 395(10227): P912-920. doi: 10.1016/S0140-6736(20)30460-8.

9. Félix-Castro JM. De pandemias, salud emocional y humanismo clínico. Rev Mex Pediatr. 2020; 87(1): 3-6. doi: 10.35366/93260. 